\title{
Ifosfamide-induced encephalopathy: about I 5 cases and review of the literature
}

\begin{abstract}
During Ifosfamide treatment, encephalopathy may occur in about $3 \%$ of cases. Additional investigations, such as biology or imaging, are usually normal. Encephalopathy is generally reversible without medium- or long-term sequelae but rare cases of death are described. It is recommended, as soon as Ifosfamide encephalopathy is suspected, to suspend its administration, to correct any hydroelectrolytic disorder, and to inject methylene blue at a dose of $50 \mathrm{mg}$ every 4 hours until the symptoms regress. Reintroduction of Ifosfamide in subsequent courses of treatment should be discussed on a case-by-case basis, under prophylactic treatment with methylene blue at a dose of $50 \mathrm{mg}$ every 6 hours. The mechanism of this complication is not fully elucidated. It is important to continue pharmacological studies in order to determine precisely the toxic metabolite(s) responsible and thus to look for an effective protector.
\end{abstract}

Volume II Issue 6 - 2020

\author{
Filali N, Elmouhtadi S, Daoudi S, Abahssain H, \\ Bechar $\mathrm{H}$, Errihani $\mathrm{H}$ \\ Department of Oncology, National Institute of Oncology, \\ Morocco
}

Correspondence: Filali N, Department of Oncology, National Institute of Oncology, Rabat, Morocco,

Email nadiafilali08@gmail.com

Received: October 23, 2020 | Published: December 31, 2020

\section{Introduction}

A structural analog of cyclophosphamide-Endoxan ${ }^{\circledR}$, ifosfamideHoloxan ${ }^{\circledR}$ is an oxazaphosphorus alkylating agent derived from nitrogen mustard.

Ifosfamide has a broad spectrum of anti-tumor activity, particularly in sarcomas (soft tissue sarcomas, osteosarcomas). The main toxicity of ifosfamide is urological but it can be prevented by a bladder protector, mesna- Uromitexan ${ }^{\circledR}$. Initially described by Van Dyck et al. ${ }^{1}$ the neurological toxicity of ifosfamide is specific and not encountered with Cyclophosphamide and Mafosfamide. This neurotoxicity, whose incidence can reach $30 \%$ according to data in the literature, seems to correspond to metabolic encephalopathy of variable severity whose etiopathogeny remains unclear.

We report the experience of the Medical Oncology Department of the National Institute of Oncology in Rabat by describing a series of cases of patients hospitalized in our department for encephalopathy with Ifosfamide. Based on fifteen observations and a review of the literature on cases of Ifosfamide encephalopathy, we attempt to specify the physiopathogeny, predisposing factors, and curative and preventive therapeutic recommendations.

\section{Patients and methods}

We report 15 cases of ifosfamide encephalopathy reported by the Medical Oncology Department of the National Institute of Oncology in Rabat over a two-year period from June 2016 to June 2018 using the notification forms provided by the National Pharmacovigilance Center. The number of patients treated with ifosfamide during this period cannot be estimated, but it can be estimated to be more than 100.

For each patient, the clinical data collected were as follows: age, sex, tumor pathology (histology, location), chemotherapy administration modalities, adjuvant treatment, number of cures, time of onset and disappearance of neurological symptomatology during the cure, treatment with methylene blue, and reintroduction of ifosfamide.

Imaging results were reported in all our patients. The neurological symptomatology was assessed according to the international classification of the National Cancer Institute (NCI).

\section{Results}

The prevalence of this neurological toxicity is probably less than $3 \%$.The age of these patients ranged from 42 to 55 years, and a large female predominance was observed with a sex ratio of 6/9. The nature of the primary tumor was variable: osteosarcoma $(n=5)$, rhabdomyosarcoma $(n=1)$, Ewing's tumor $(n=3)$, synovialosarcoma $(\mathrm{n}=1)$ and Hodgkin's and non-Hodgkin's lymphoma $(\mathrm{n}=4)$. In one patient, the primary tumor was localized to the pelvis.

Ifosfamide was administered, in the same manner in all patients, as a 3-hour intravenous infusion in combination with parenteral hydration on the basis of $2 \mathrm{~L} / \mathrm{m}^{2}$ per day and uro protection with Mesna, at a dosage of $1.8 \mathrm{mg} / \mathrm{m} 2$ per course for 3-5 days, in combination with other cytotoxic drugs.

National Cancer Institute (NCI) grade II and grade III neurological toxicity was observed in all 15 patients within 24-72 hours of Ifosfamide administration resulting in discontinuation of the current infusion. It was marked primarily by somnolence, agitation, confusion and hallucinations. A brain CT scan was performed in all patients and returned to normal. All patients were treated with methylene blue. The evolution was favorable in 13 patients and unfavorable in two patients who died following complications of encephalopathy and whose hospitalization lasted 15 days.

The imputability study conducted at the National Pharmacovigilance Center is ongoing. In addition, a letter from the Poison Control Center was sent to us to inform us of the new recommendations for the conservation of Ifosfamide due to the risk of encephalopathy.

\section{Discussion}

Ifosfamide encephalopathy is a rare event during discontinuous slow infusion parenteral therapy. In our experience, the number of patients treated with Ifosfamide during this period cannot be estimated, but it can be estimated to be over 100 , therefore.

The prevalence of this neurological toxicity is probably less than $3 \%$. The incidence reported in the literature ranges from $0 \%$ to more than $30 \%{ }^{2,3}$ Most teams find a low incidence $(<3 \%)$, however, some teams report $22 \%$ acute neurotoxicity in patients receiving this treatment. ${ }^{4}$ A high incidence (30\%) of severe neurological disorders (confusion, convulsion, coma) has been described with ifosfamide during the treatment of pediatric brain tumors. ${ }^{4}$ 
This neurotoxicity affects adults as well as children, but is more severe in children. ${ }^{5-7}$ In our short series, there is a clear predominance of females; no difference between the sexes is found in the literature. ${ }^{5,8}$

The ifosfamide administration regimen appears to play a role in the occurrence of neurotoxicity. In the Curtin et al. study, ifosfamide was administered in a variable manner: when administered discontinuously for 5 days in a row at a dose of 1.2 to $1.5 \mathrm{~g} / \mathrm{m}^{2}$ per day, no patient experienced grade IV neurotoxicity, whereas in patients who received ifosfamide continuously over 24 hours at a dose of 2.5 to $5 \mathrm{~g} / \mathrm{m}^{2}$ per day, 33\% experienced grade IV neurotoxicity. ${ }^{9}$ The neurological toxicity of ifosfamide appears to be significantly more frequent with continuous bolus or intravenous administration $(p<0.001))^{2,10,11}$ In the study by Cerny et al. ${ }^{10}$ oral administration was associated with a high incidence of encephalopathy. Thus, the risk of neurotoxicity appears to be significantly associated with ifosfamide administration over 15 minutes, 1 hour, ${ }^{6}$ or continuously over 24 hours. ${ }^{12}$ According to Perren et al. ${ }^{11}$ a short administration time of less than 6 hours may increase the likelihood of developing encephalopathy by probably increasing CNS concentrations of ifosfamide. Similarly, neurotoxicity appears more frequent with a single dose of ifosfamide compared to a dose divided over 1 to 5 days. ${ }^{2,12,13}$ Some authors have also invoked the responsibility of psychotropic drugs and antiemetics administered concomitantly in high doses. ${ }^{5}$ However, the observation of encephalopathy outside of this type of parallel treatment only allows us to attribute to them an accessory and little contributing role., ${ }^{5,13}$ The rank of the ifosfamide treatment during which neurotoxicity occurs is highly variable in our series, indicating a probable individual predisposition. Similarly, during the course of the treatment, the time of onset of neurological symptoms varied from a few hours to 72 hours. In the study by Merimsky et al. ${ }^{16}$ in 28 adult patients who received Ifosfamide, 5 patients $(17.8 \%)$ had encephalopathy with onset of symptoms between days 2 and 7. In our series, all of our patients presented with grade II or III Ifosfamide neurological toxicity. Pratt et al..$^{5}$ observed mainly central neurological signs (behavioral changes, cerebellar syndrome, urinary incontinence, convulsions, coma, excessive drowsiness), but also peripheral manifestations (transient deafness, facial paralysis). In the study by Rieger et al. ${ }^{17}$ in 60 patients who received Ifosfamide, 17 patients developed neurological signs: grade I in 4 cases, grade II in 2, grade III in 7 and grade IV in 4 cases. In our series, somnolence regressed in 24 to 36 hours (median 72 hours). We observed two deaths. The central toxicity of Ifosfamide is usually completely reversible and may persist for hours to days, typically 3 to 5 days after the last administration..$^{5,12,18}$

The results of blood biologic tests (electrolytes, serum calcium, magnesemia, blood gas) were normal.,19 No morphological abnormalities were noted in our patients. Brain imaging by computed tomography (CT) scan or MRI is most often normal. ${ }^{19}$ Morphological studies by brain CT scan show no correlation between the severity of encephalopathy and the size of the ventricles. ${ }^{16,20,21}$

According to various studies, the EEG shows only diffuse abnormalities of non-specific cortical pain, suggesting a toxictype encephalopathy. ${ }^{5,13,14}$ There is a relationship between EEG abnormalities and the degree of severity of clinical neurological toxicity. ${ }^{13,22}$

The specific neurotoxicity of Ifosfamide could correspond to an encephalopathy by accumulation of toxic metabolites, which remain to be formally identified. Like its structural analogue, Cyclophosphamide, Ifosfamide is a prodrug whose activation is mediated by the hepatic mixed-function microsomal system, cytochrome $\mathrm{P} 450 .{ }^{23}$ Cyclophosphamide, even at very high doses, does not expose to neurotoxicity. Thus, it can be hypothesized that it is the metabolites that differ between Cyclophosphamide and Ifosfamide that may be involved. The hydroxylation of Ifosfamide is much slower than that of its analogue, allowing other metabolic pathways to be involved. ${ }^{24}$ The result is a 10 -fold increase in the concentration of chloroacetaldehyde. ${ }^{25}$ Chloroacetaldehyde is a neurotoxic substance $^{26}$ and its role is incriminated by some authors ${ }^{24,27}$ and controversial by others. ${ }^{10,25}$ Encephalopathy could be due to inhibition of the metabolism of long-chain fatty acids by chloroacetaldehyde. ${ }^{27}$ Numerous studies have shown the efficacy of Methylene Blue in the treatment of encephalopathies with Ifosfamide..$^{26,28-32}$ Methylene Blue could act at different levels: inhibition of flavoproteins in the respiratory chain of mitochondria, ${ }^{29}$ reoxidation of NADH and hepatic glucose production, ${ }^{33}$ inhibition of monoamine oxidase, thus preventing the formation of chloroacetaldehyde. ${ }^{29,34}$ On the other hand, Methylene Blue has no interaction with the alkylating agents of Ifosfamide and therefore does not reduce the antitumor efficacy of the treatment. ${ }^{34}$ Some authors have been able to correlate the incidence of encephalopathies in adults and children with certain parameters such as advanced age, ${ }^{13}$ pelvic tumor location, ${ }^{14}$ previous nephrectomy,,${ }^{35}$ and prior Cisplatin treatment. ${ }^{5,36}$ Pediatric forms are generally more severe than those in adults. ${ }^{5-7}$ Biologically, a decrease in creatinine clearance $^{13}$ and hypoalbuminemia ${ }^{9,36,37}$ have also been presented as risk factors for ifosfamide encephalopathy. In our series, none of the described risk factors were found. The recent study by Rieger et al. ${ }^{17}$ could not identify any risk factors and the authors put forward the notion of an individual predisposition. Concerning the risk of recurrence during readmission, controversy persists. Several teams report no recurrence of symptoms upon re-administration. ${ }^{5,36,38}$ Pratt et al. ${ }^{5}$ investigated whether patients who developed neurotoxicity were at increased risk of encephalopathy in 65 patients who received more than 2 courses of Ifosfamide; 10 patients developed neurotoxicity, and for these 10, the same symptoms reappeared in the following courses. A total of 15 cures were administered in these 10 patients: 13 were followed by neurotoxicity of varying degrees. There was no significant increase in the severity of neurological toxicity between the first and subsequent episodes in this series; however, other authors have reported several cases of severe or even fatal progression upon reintroduction of Ifosfamide. ${ }^{7,12,19,39}$

The occurrence of Ifosfamide encephalopathy is unpredictable, but caution should be exercised when using this antimitotic in patients with these risk factors. In our series, Methylene Blue was administered to all patients.

The use of Methylene Blue is recommended for the regression of neurological disorders. Küpfer et al. ${ }^{30}$ were the first authors to suggest the efficacy of Methylene Blue in encephalopathy related to Ifosfamide administration. The authors hypothesized that this product compensates for the inhibition of glycogenesis caused by the inhibition of fatty acid oxidation. They reported the observation of a patient who became somnolent a few hours after the start of Ifosfamide treatment and in whom treatment with Methylene Blue at a dosage of $50 \mathrm{mg}$ as a 30-minute intravenous infusion was followed by a return to normal consciousness within 4 hours. This patient's urine analysis showed excess glutaric acid and sarcosine, as usually seen in glutaryl CoA dehydrogenase enzyme deficiency (type I) and flavoprotein deficiency (type II). Methylene Blue is successfully used in the treatment of Type I glutaric aciduria by acting as an electron acceptor and restoring the activity of glutaryl CoA dehydrogenase. Aeschlimann et al. ${ }^{34}$ evaluated the efficacy of Methylene Blue as a neuroprotective agent: 
out of 52 patients treated with Ifosfamide, 12 had a grade II or higher $\mathrm{NCI}$ neurotoxicity. Eight patients were treated with Methylene Blue at a dose of $50 \mathrm{mg}$ every 4 hours intravenously upon clinical diagnosis of encephalopathy. In 1 out of 2 cases, neurological signs disappeared within 24 hours, 3 of which were before 12 hours and in 1 case 1 hour after administration of Methylene Blue. In the 2 patients who did not receive Methylene Blue, reversibility was observed after 72 hours. ${ }^{26}$ Other sporadic observations confirmed the efficacy of Methylene Blue. ${ }^{20,28,31,32,40}$ In the case of Ifosfamide-induced encephalopathy, it seems justified to discontinue Ifosfamide and to administer Methylene Blue. The dosage used by all authors is $50 \mathrm{mg}$ every 4 hours intravenously until complete regression of symptoms. Several authors report observations suggesting the efficacy of WB in the prevention of recurrence of neurological disorders..$^{20,31,41}$ In the study by Pelgrims et al. ${ }^{3}$ patients who received Methylene Blue as a preventive measure in the same way as before, 2 had a lower grade of neurotoxicity. ${ }^{26}$ In patients who have experienced neurotoxicity with Ifosfamide, Turner et al. therefore recommend continuing Ifosfamide in combination with Methylene Blue at a dose of $50 \mathrm{mg}$ intravenously every 6 hours throughout the course of treatment. ${ }^{20}$

\section{Conclusion}

During Ifosfamide treatment, encephalopathy may occur in about $3 \%$ of cases. Additional investigations, such as biology or imaging, are usually normal. Encephalopathy is generally reversible without medium- or long-term sequelae but rare cases of death are described. It is recommended, as soon as Ifosfamide encephalopathy is suspected, to suspend its administration, to correct any hydroelectrolytic disorder, and to inject methylene blue at a dose of $50 \mathrm{mg}$ every 4 hours until the symptoms regress. Reintroduction of Ifosfamide in subsequent courses of treatment should be discussed on a case-bycase basis, under prophylactic treatment with methylene blue at a dose of $50 \mathrm{mg}$ every 6 hours. The mechanism of this complication is not fully elucidated. It is important to continue pharmacological studies in order to determine precisely the toxic metabolite(s) responsible and thus to look for an effective protector.

\section{Acknowledgments}

None.

\section{Conflicts of interest}

The authors declare there are no conflicts of interest.

\section{Funding}

None.

\section{References}

1. Van Dyck JJ, Falkson HC, Van der Merwe AM, et al. Unexpected toxicity in patients treated with ifosfamide. Cancer Res. 1972;32(5):921-924.

2. Elias AD, Eder JP, Shea T, et al. High dose ifosfamide with mesna uroprotection: a phase I study. J Clin Oncol. 1990;8(1):170-178.

3. Brade WP, Herdrich K, Varini M. Ifosfamide pharmacology, safety and therapeutic potential. Cancer Treat Rev. 1985;12(1):1-47.

4. Orbach D, Brisse H, Doz F. Central neurological manifestations during chemotherapy in children. Arch Pediatr. 2003;10(6):533-539.

5. Pratt $\mathrm{CB}$, Green AA, Horowitz ME, et al. Central nervous system toxicity following the treatment of pediatric patients with ifosfamide/ mesna. J Clin Oncol. 1986;4(8):1253-1261.
6. Gieron MA, Barak LS, Estrada J. Severe encephalopathy associated with ifosfamide administration in two children with metastatic tumors. $J$ Neurooncol. 1988;6(1):29-30.

7. Verdeguer A, Castel V, Esquembre C, et al. Fatal encephalopathy with ifosfamide/mesna. Pediatr Hematol Oncol. 1989;6(4):383-385.

8. Kellie S, Pritchard J, Bowman A. Ifosfamide neurotoxicity in children. $J$ Clin Oncol. 1987;5(3):512-514.

9. Curtin JP, Koonings PP, Gutierrez M, et al. Ifosfamide induced neurotoxicity. Gynecol Oncol. 1991;42(3):193-196.

10. Cerny T, Castiglione M, Brunner K, et al. Ifosfamide by continuous infusion to prevent encephalopathy. Lancet. 1990;335(8682):175.

11. Perren TJ, Turner RC, Smith IE. Encephalopathy with rapid infusion ifosfamide/mesna. Lancet. 1987;1(8529):390-391.

12. Watkin SW, Husband DJ, Green JA, et al. Ifosfamide encephalopathy: a reappraisal. Eur J Cancer Clin Oncol. 1989;25(9):1303-1310.

13. Meanwell CA, Blake AE, Kelly KA, et al. Prediction of ifosfamidemesna associated encephalopathy. Eur $J$ Cancer Clin Oncol. 1986;22(7):815-819.

14. Meanwell CA, Blake AE, Latief TN, et al. Encephalopathy associated with ifosphamide-mesna therapy. Lancet. 1985;1(8425):406-407.

15. Cerny T, Kupfer A. The enigma of ifosfamide encephalopathy. Ann Oncol. 1992;3(9):679-681.

16. Merimsky O, Reider-Groswasser I, Wigler N, et al. Encephalopathy in ifosfamide-treated patients. Acta Neurol Scand. 1992;86(5):521-525.

17. Rieger C, Fiegl M, Tischer J, et al. Incidence and severity of ifosfamideinduced encephalopathy. Anticancer Drugs. 2004;15:347350

18. Cantwell BM, Harris AL. Ifosfamide-mesa and encephalopathy. Lancet. $1985 ; 1(8431): 752$

19. Antoine E, Bernat I, Feuvret L, et al. Encéphalopathies à l'Holoxan. Cah Oncol. 1993;2:103-109.

20. Turner AR, Duong CD, Good DJ. Methylene blue for the treatment and prophylaxis of ifosfamide-induced encephalopathy. Clin Oncol (R Coll Radiol). 2003;15(7):435-439.

21. Merimsky O, Inbar M, Reider-Groswasser I, et al. Ifosfamide-related acute encephalopathy: clinical and radiological aspects. Eur J Cancer. 1991;27(9):1188-1189.

22. Wengs WJ, Talwar D, Bernard J. Ifosfamide-induced nonconvulsive status epilepticus. Arch Neurol. 1993;50(10):1104-1105.

23. Dechant KL, Brogden RN, Pilkington T, et al. Ifosfamide-mesna. A review of its antineoplastic activity, pharmacokinetic properties and therapeutic efficacy in cancer. Drugs. 1991;42(3):428-467.

24. Goren MP, Wright RK, Pratt CB, et al. Dechloroethylation of ifosfamide and neurotoxicity. Lancet. 1986;2(8517):1219-1220.

25. Kerbusch T, de Kraker J, Keizer HJ, et al. Clinical pharmacokinetics and pharmacodynamics of ifosfamide and its metabolites. Clin Pharmacokinet. 2001;40(1):41-62.

26. Pelgrims J, De Vos F, Van den Brande J, et al. Methylene blue in the treatment and prevention of ifosfamide-induced encephalopathy: report of 12 cases and a review of the literature. Br J Cancer. 2000;82(2):291294.

27. Visarius TM, Stucki JW, Lauterburg BH. Inhibition and stimulation of long-chain fatty acid oxidation by chloroacetaldehyde and methylene blue in rats. J Pharmacol Exp Ther. 1999;289(2):820-824. 
28. Zulian GB, Tullen E, Maton B. Methylene blue for ifosfamide-associated encephalopathy. N Engl J Med. 1995;332(18):1239-1240.

29. Kupfer A, Aeschlimann C, Cerny T. Methylene blue and the neurotoxic mechanisms of ifosfamide encephalopathy. Eur J Clin Pharmacol. 1996;50(4):249-252.

30. Kupfer A, Aeschlimann C, Wermuth B, et al. Prophylaxis and reversal of ifosfamide encephalopathy with methylene-blue. Lancet. 1994;343(8900):763-764.

31. Ferrero JM, Eftekari P, Largillier R, et al. Traitement de l'encéphalopathie à l'Ifosfamide par le bleu de méthylène. Bull Cancer. 1995;82:598-599.

32. Alonso JL, Nieto Y, Lopez JA, et al. Ifosfamide encephalopathy and methylene-blue: a case report. Ann Oncol. 1996;7(6):643-644.

33. Greenberg SR, Kerr JH. Methylene blue in alcohol-induced hypoglycaemia. Lancet. 1965;2(7422):1132.

34. Aeschlimann C, Cerny T, Kupfer A. Inhibition of (mono)amine oxidase activity and prevention of ifosfamide encephalopathy by methylene blue. Drug Metab Dispos. 1996;24(12):1336-1339.

35. Heim ME, Fiene R, Schick E, et al. Central nervous system effect following ifosfamide monotherapy of advanced renal carcinoma. $J$ Cancer Res Clin Oncol. 1981;100(1):113-116.
36. Pratt CB, Goren MP, Meyer WH, et al. Ifosfamide neurotoxicity is related to previous cisplatin treatment for pediatric solid tumors. J Clin Oncol. 1990;8(8):1399-1401.

37. Meanwell CA, Kelly KA, Blackledge G. Avoiding ifosfamide-mesna encephalopathy. Lancet. 1986;2(8503):406.

38. Antman KH, Ryan L, Elias A, et al. Response to ifosfamide and mesna: 124 previously treated patients with metatastic or unresectable sarcoma. J Clin Oncol. 1989;7(1):126-131.

39. McCallum AK. Ifosfamide-mesna encephalopathy. Lancet. 1987;1(8539):987.

40. Raj AB, Bertolone SJ, Jaffe N. Methylene blue reversal of ifosfamiderelated encephalopathy. $J$ Pediatr Hematol Oncol. 2004;26(2):116.

41. Kasper B, Harter C, Meissner J, et al. Prophylactic treatment of known ifosfamide- induced encephalopathy for chemotherapy with high-dose ifosfamide? Support Care Cancer. 2004;12(3):205-207. 